\title{
Wireless LED Dimming Control Based on WiFi
}

\author{
BaoJianyu ${ }^{1, \text { a }}$, Zhang Shan ${ }^{2, b}$ \\ ${ }^{1}$ Ningbo Institute of Technology, Zhejiang University, Ningbo, Zhejiang, China; \\ ${ }^{2}$ Taiyuan University of Science and Technology, Taiyuan, China \\ aemail:jianyu_bao@163.com
}

Keywords: LED; LED dimming; WiFi; STC MCU; wireless communication

\begin{abstract}
To fit for the development of the personal needs for light-emitting diode (LED) lighting, a wireless LED dimming controller based on WiFi module is presented in this paper. Low-cost 8-bit STC MCU is applied as the main controller,WiFi is used as the wireless communication module, which receives the control signals from MCU through serial ports. Through WiFi communication between LED dimmer and mobile phone, the APP software installed on mobile phone terminal is used for wirelessly adjusting the brightness, color or color temperature of LED lamps.
\end{abstract}

\section{Introduction}

As a new type of solid-state lighting source, LED has the advantages of high efficiency, low energy consumption, sturdiness and durability, long service life, good safety, and has been a hot research in the lighting industry[1]. Dimming control of LED lighting can conserve energyfurtherly. LED dimming control system based on wire communication has shortcomings suchas complex wiring, poor scalability, high cost of installation and maintenance and poor mobility. Therefore, it may be agood choice to use wireless communication technique for LED intelligent dimming. With the fast development of WiFi technology,smart mobile phonewith WiFihas been widely used at those occasions such as office and home for remote control. Besides, hardware and software updates constantly, signal becomes more and more stable, wireless applications are also increasingly widespread. Compared with bluetoothdimming, WiFidimming has a wider range coverage and higher transmission velocity, and it also has a stronger feeling of science. Therefore, it is meaningful to investigate LED dimming control by using WiFi technique. In this paper, Wireless LEDdimming technique based on mobile phone WiFi will be emphatically discussed.

\section{Operational principle}

The block diagram of dimming controlwith WiFiis shown in Fig.1,the system consists of LED driver with PWM interface, WiFi wireless network and a MCU for system control.

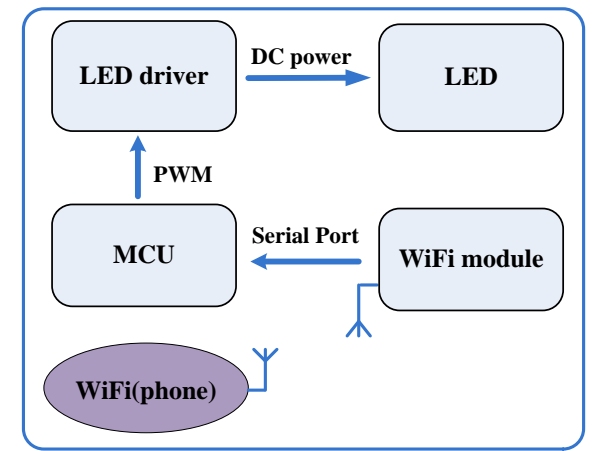

Fig.1: The block diagram of dimming control

To effectively control LED lighting, APP software should be installed in advance on mobile phone terminal, and thus a smart mobile phone can simultaneously control multiple sets of LED lamps by sending dimming control signal through APP. An independentWiFi module is embedded in the lamp as a receiving moduleto communicate with the WiFimodule from mobile phone. When the lamp WiFi receivesthe dimming control signals from mobile phone,and then these signals will 
be transmitted to MCU controller through the serial ports.By decoding and modulation process, MCU generates the corresponding PWM signalsto LED driver, and thus those functions such as on/off control, brightness and color adjustment, can all be implemented on the mobile phone terminal.

\section{Hardware circuit design}

STC89C52RC[3] is selected as MCU controller, WIFI232-G is a low power embedded WiFi module, integrated with MAC, baseband chip, RF transceiver unit, and power amplifier.

The LED driver circuit[5], is shown in Fig. 2.PT4115 is used as the main chip[6], which is a buck constant current control IC operating in continuous inductor current conduction mode, and can be used to drive one or more series LED. Output current of PT4115 can be adjusted,the maximum value can be up to 1.2A. This chip supports PWM dimming, and the pulse width modulation signal is converted to the current signal with different duty cycle by the buck circuit, and thus to achieve PWM dimming for LED lighting.

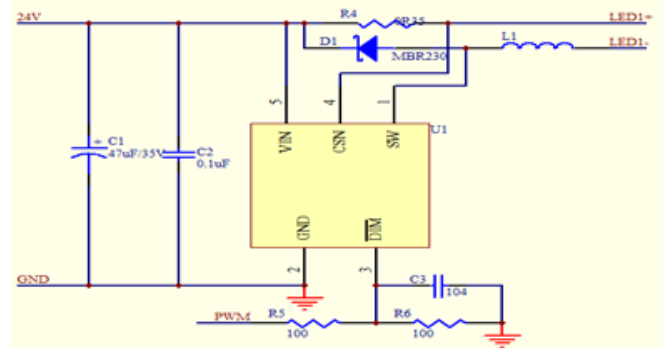

Fig.2: LED driving circuit based on PT4115

The main peripheral device parameters arecalculatedaccording to the data sheet and the output requirements of PT4115.

Sampling resistor $R_{4}$, control the size of the output current, it determines the absolute accuracy of the constant current source. The value of $R_{4}$ is related to the size of the load current, generally with the following formula:

$$
I_{\text {out }}=\frac{0.085+0.115}{2 \times R_{4}}=\frac{0.1}{R_{4}}
$$

As the load is 5 series $1 \mathrm{~W}$ white LED, the rated current is $350 \mathrm{~mA}$, the value of the sampling resistor $\mathrm{R} 4$ is:

$$
R_{4}=\frac{0.1}{0.35}=0.2857 \Omega
$$

$L_{1}$ is a rectification inductance, is a key element in this circuit, its function is to transform the high-frequency pulse current into a triangular-wave current. The inductance of $L_{1}$ will affect the stability of the constant current source within the operating voltage range. Because the best operating frequency of PT4115 is below $1 \mathrm{MHz}$, small inductance will affect its operating frequency, the inductance should be above 68uH.So the operating frequency of the system can be controlled to be under $1 \mathrm{MHz}$. The smaller inductance, the higher working frequency. As the response speed limit of the internal current detection circuit, it has effects on internal current detection, and thus the internal switch on/off control can't be well implemented. In addition, too small inductance may be cause the damage of SW. Therefore, inductance should be selected as $100 \mathrm{uH}$.

$D_{1}$ is a freewheeling diode, which provides a discharge circuit for the current stored in the inductor when the MOSFET is in the off state. Due to the high frequency operation, $D_{1}$ should be selected as Schottky diode whose forward voltage drop is small, and its recovery time is very short. According to the requirements, ONSEMI Company's MBR230 is selected.

\section{Software design}

Software design includes the main program design, serial communication program design and PWM generation program design. Fig. 3(a) shows how to implement the communication between 
WiFi module and MCUthrough serial port.If the serial interruption is trigged, the lampWiFimodule will begin to receive data from mobile phone, and the received data will be saved for generating PWM signals to LED dimming.Then, by continuousinquiry of the serial interruption in the main program, the data of duty-cycle will be transferred to PWM generation sub-program as shown in Fig. 3(b).

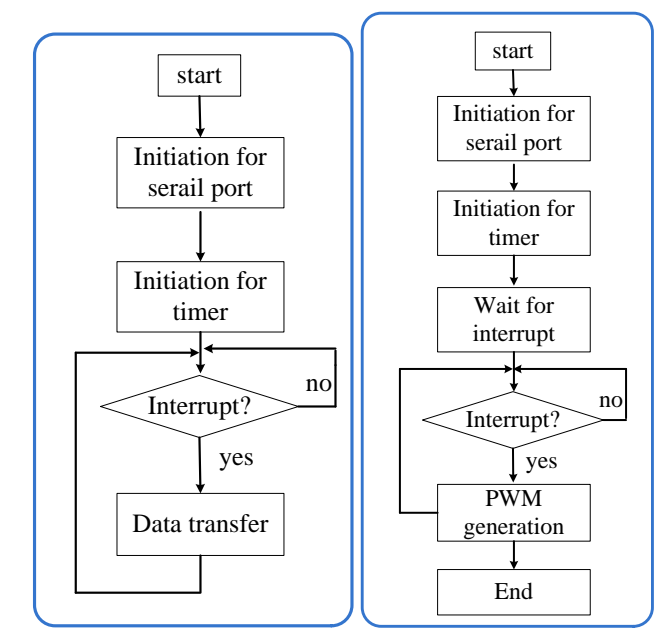

(a)Serial communication program(b) System program

Fig.3: Flow chartdiagram

PWM generationprogram is the key part of the whole system, which is finished in MCU. Before generating PWM signals, MCU must deal with the mobile phone's instructions, which contains a command or protocolto determine and perform different actions depending on the instruction content action. In this design,the protocol is defined as follows: when MCU receives hexadecimal "03"from mobile phone,it outputs 100\% duty-cycle PWM waveform.IfMCU receives hexadecimal "04", it outputs $0 \%$ duty-cycle PWM waveform. While if hexadecimal "01"is received, the duty-cycle of PWM waveform will increasecontinuously until 100\%; when hexadecimal "02"is received, the duty-cycle of PWM waveform willdecreasecontinuously until $0 \%$.Thus, the dimming instructions from mobile phone are transformed into PWM signals to LED driver by MCU decoding.

\section{Experimental results}

To testify this proposed scheme, a 5W LED lighting prototype is built. The whole experimental system consists ofandroid mobile phone, USR-WIFI232-G module, MCU control board based on STC89C52RC, LED driver circuit based on PT4115, oscilloscope, LED light source (5W/350mA). UDP communication software is installed in an android mobile phone. The control command is sent through UDP communication software, different hexadecimal numbersare sent to LED driver to obtain different duty cycles to adjust the brightness of the LED lamp in the experimental system.

When sending hexadecimal "01" from mobile phone APP, MCU generates the corresponding PWM waveform with $10 \%$ duty cycle, LED lamp is lighting but its brightness is dark, whichis shown in Fig.4. If continuously transmitting hexadecimal "01"to LED driver, the corresponding duty cycle of PWM waveform will also continuously increase. Fig.5 and Fig.6 show the corresponding PWM waveforms with about 50\% and 90\% duty cycle respectively,with the increase of duty cycle, LED lamp continues to light and its brightness gradually become strong.

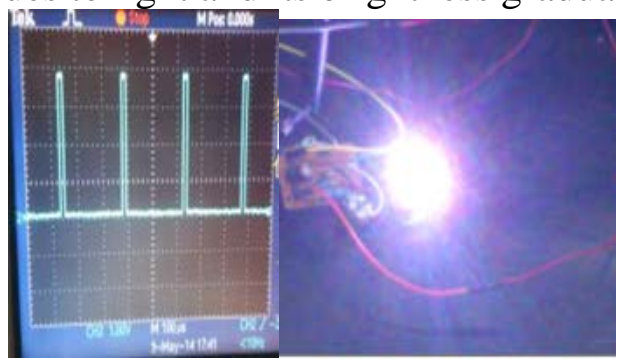

(a)10\% duty cycle PWM waveform(b) LED lamp brightness 
Fig.4: The waveform of $10 \%$ duty cycle when sending "01"

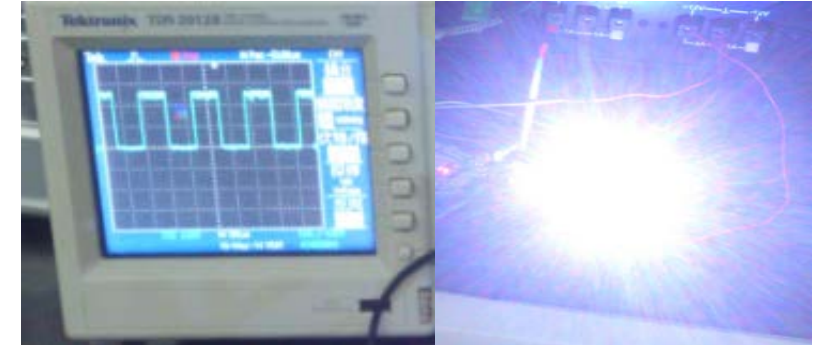

(a) 50\% duty cycle PWM waveform

(b) LED lamp brightness

Fig.5: The waveform of 50\% duty cycle when sending "02"

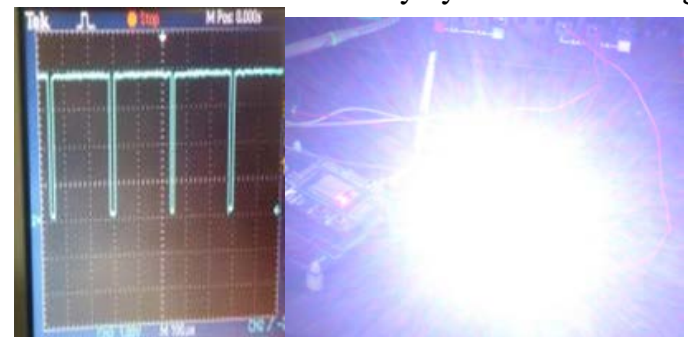

(a) $90 \%$ duty cycle PWM waveform

(b) LED lamp brightness

Fig.6: The waveform of 50\% duty cycle when sending "03"

\section{Conclusion}

The traditional LED dimming control system has shortcomings such as complex wiring, poor scalability, low energy saving efficiency etc. In this paper, LED wireless dimming control is achieved by mobilephone WiFi technique, this wireless system can not only switch LED lamp on or off, but also adjust its brightness and color. The expected results areachieved. Controlling LED lamp by mobile phones connected with $\mathrm{WiFi}$, solves the inconvenience and uncontrollable brightness problems of traditional switches. Therefore, it is meaningful to control LED lighting through Wi-Fi technique.

\section{Acknowledgment}

Thisresearch work was supported by LED Support Project of Ningbo,through grant number 2014 B92003.

\section{References}

[1] Mao Xingwu, ZhangYanwen, ZhouJianjun, ZhuDawei. A new generation of LED green light source and its application technique.Beijing: People's Posts and Telecommunications Press, 2008.

[2] Wang Juan, GuoJiaqi, Liu Wei.Deep discussion and Research on WIFI technology.Value Engineering, 30 (6) : 91-92. 2011.

[3] STC89C52RC Series Single Chip Machine guidence sheet. Macro crystal technology.

[4]USR-WIFI232-G low power embedded WiFi module user manual. Ji'nan USR IOT technology Co., Ltd..

[5]Zhang Haocheng. LED dimming scheme and its driver design. Xi'an: Xi'an Electronic and Science University, 2012.

[6] PT4115_DS Rev CH_2.6.Information on http:// WWW.CRPOWTECH.COM. 